\title{
Open Access in India and Way Forward for South Asia
}

Access to information is the first step for the advancement and progress of the society. And the Open Access, which is immediate availability of the scientific literature and other research outputs upon publication required for the advancement and progress of the science. The Open Access movement in the South Asian region comprising Afghanistan, Bangladesh, Bhutan, India, Nepal, Maldives, Pakistan and Sri Lanka is skewed with more awareness and participation in India and with no or nil in Afghanistan and Maldives. When we look at the level of access to information to scientific information in South Asian region through the Open Access Repositories, it's only about 140 repositories registered with the ROAR while in the world, 4661 repositories are registered. The registry of Open Access mandates shows that only 17 (with one mandate from Pakistan) mandates are registered with the database from the South Asian region while a total of 941 mandates were registered from around the world. The Directory of Open Access Journals (DOAJ) had registered 12026 journals published from 121 countries. The INASP's JOL project is facilitating hosting of some of the journals from Asia viz., Bangladesh Journals Online (15670 Records), Nepal Journals Online (14372 Records) and Sri Lanka Journals Online (10082 Records) and is providing access to all of them via harvester, Asian Journals Online.

A year after the Budapest Open Access Initiative (BOAI) was released in 2002, the seeds of the Open Access movement were sown in India. However, the momentum had not gained as it has should have been in the country and in the region, South Asia when compared to Europe and Latin America. In the recent past, with the initiatives of the individuals, communities of practice, institutes and the funding agencies (both private and public) there was an acceleration in the progress of Open Access. The countries, especially in South Asia viz., Bangladesh, India, Nepal, Pakistan, Sri Lanka etc., have now communities of practice advocating for Open Access to the public funded research. And this region now has policies and infrastructures in place for opening up access to the public funded research. For example, in India, the Department of Biotechnology and Department of Science and Technology, Government of India, Council of Scientific and Industrial Research, Indian Council of Agricultural Research has adopted policies for Open Access and had also made available suitable infrastructure. It is now mandatory for all the doctoral degree scholars to deposit their thesis as per the directive of the Ministry of Human Resources Development, Government of India. Efforts are also being made to deliver all the openly available resources through a single platform called National Digital Library of India. However, there is no mandatory national policy formulated by any of the countries in South Asia. 
While there is no problem in deposition of the whole or part of the thesis by the scholars in a publicly available repository, the deposition of journal articles along with or without data in public repositories has had some conflict. Still, researchers and the research managers of the institutes or universities are not clear about the legal issues of sharing the research outputs immediately. There is a very low filling of the repositories with fulltexts in India even when there is a policy and infrastructure in place. The possible reasons for the low populating rate of articles in the repositories is that though the policies are in place, there are no rewarding/incentive mechanisms for openly sharing the research outputs and due to copyright transfer agreements. And the research, evaluation and monitoring committees in the universities or institutes solely depend upon publications in the journal impact factors. As availability of the relevant journals with higher impact factors from the South Asian countries are limited in numbers and also have a high rejection rate. By publicizing fake metrics, large number of questionable journals are coming up and are luring the scholars to submit articles to their publications. These journals are questionable journals which publish without any review, but for a charge. The scholars are taking this road in order to publish in numbers and in no time. Though the availability of quality articles via repositories is less, the availability of same via open access journals is much more.

The scholarly societies in India, though trying to catch up with the technology due to the traditional character of publication in print journals, there is a huge time lag from submission to publication. Some of the societies are collaborating with the commercial for profit publishers for online publications and are not able to provide immediate free access to the published articles due to embargo. The efforts by the University Grants Commission for building a white list of journals and the rating of the journals by another academy can be seen as quality control mechanisms in scholarly publications. But more efforts are needed for making Open Access as default by 2025. The DOAJ ambassadors in India are also working with the applications for indexing and are also raising the awareness on Quality Open Access Journals. To put forth the alternate pathway for sharing the research outputs for public to read and review it as soon as possible and also to cut short the long time gap in the publication process while giving the priority of work published, preprint repositories are becoming popular and gaining importance in the scholarly communication process across all the disciplines which was earlier confined to physics only. The Open Access India a community of practice advocating for Open Access, Open Data and Open Education, had launched a preprint repository exclusively for agriculture and allied sciences, AgriXiv with the help of the Centre for Open Science. Now the Open Access India is planning to launch IndiaRxiv for all the disciplines and for all the scholarly outputs for the Indian researchers and scholarship.

Recently the advocates and practitioners of Open Access in India had pounced 'Delhi Declaration on Open Access' to commemorate the BOAI which was adopted sixteen years ago. The signatories of the declaration were not only of India, but the other countries in the South. This shows the concern and solidarity for the Open Access initiatives by the Global South constituents. The communities of practice in South Asia under the initiative 
of Open Access India are planning for the formation of the regional Open Access forum on the similar lines of Latin America. The South Asian region has the communities of practices: IFSMA, Open Access Bangladesh, Open Access Nepal, Open Access Pakistan, SAARC Wikimedia. And all these communities working for Open Access in this region have more lessons to learn from the Open Access movement from each other. The success stories and the strategies along with the successful research sharing, evaluation, monitoring and rewarding mechanisms are very crucial learning resources for the development of a regional cooperation for Open Access policy and infrastructure development in South Asia. There is a need for an in-depth reflection regarding global developments in Open Access and what they mean from the vantage point of the Global South, and to develop a perspective in which the agency of the people of the Global South, from historically informed ways, contributes in co-shaping the contemporary world. The session should work for the understanding and strategy and design to exchange information on advances and challenges in Open Access in South Asia, and discuss an Action Plan to Strengthen for a community-led non-commercial international Open Access future. Sharing the status and challenges of Open Access, discussing the way forward and building the Roadmap for Open Access as default by 2025 and the formation of a regional community of practice, (Forum for Open Access in South Asia) and pronouncing the South Asian Forum's Declaration on Open Access should be the outcome of the session/workshop.

Sridhar Gutam

Senior Scientist (Plant Physiology), ICAR-IIHR \& Convenor, Open Access India Bengaluru, India gutam2000@gmail.com 